\title{
Genetic influences on exercise-induced adult hippocampal neurogenesis across 12 divergent mouse strains
}

\author{
Peter J. Clark, Rachel A. Kohman, Daniel S. Miller, Tushar K. Bhattacharya, Weronika J. \\ Brzezinska, and Justin S. Rhodes \\ University of Illinois, Department of Psychology, Beckman Institute, 405 N. Mathews Ave, \\ Urbana, IL 61801
}

\begin{abstract}
New neurons are continuously born in the hippocampus of several mammalian species throughout adulthood. Adult neurogenesis represents a natural model for understanding how to grow and incorporate new nerve cells into pre-existing circuits in the brain. Finding molecules or biological pathways that increase neurogenesis has broad potential for regenerative medicine. One strategy is to identify mouse strains that display large versus small increases in neurogenesis in response to wheel running so the strains can be contrasted to find common genes or biological pathways associated with enhanced neuron formation. Therefore, mice from 12 different isogenic strains were housed with or without running wheels for 43 days to measure the genetic regulation of exercise-induced neurogenesis. The first 10 days mice received daily injections of BrdU to label dividing cells. Neurogenesis was measured as the total number of BrdU cells co-expressing NeuN mature neuronal marker in the hippocampal granule cell layer by immunohistochemistry. Exercise increased neurogenesis in all strains, but the magnitude significantly depended on genotype. Strain means for distance run on wheels, but not distance traveled in cages without wheels, were significantly correlated with strain mean level of neurogenesis. Further, certain strains displayed greater neurogenesis than others for a fixed level of running. Strain means for neurogenesis under sedentary conditions were not correlated with neurogenesis under runner conditions suggesting that different genes influence baseline versus exercise-induced neurogenesis. Genetic contributions to exercise-induced hippocampal neurogenesis suggest that it may be possible to identify genes and pathways associated with enhanced neuroplastic responses to exercise.
\end{abstract}

\section{Keywords}

adult hippocampal neurogenesis; wheel running; aerobic exercise; inbred strains; F1 hybrids; home cage activity; BrdU; heritability; genetic correlations

\section{Introduction}

The discovery that new nerve cells are continuously born in the hippocampus of adult rodents and humans has generated great interest (Deng et al. 2010; Kempermann 2002; Schinder \& Gage 2004). Adult hippocampal neurogenesis represents a natural model for understanding how to regenerate and incorporate new neurons into preexisting circuits in the adult brain. If the process could be controlled, then the resulting technology could have broad therapeutic potential for cognitive aging, neurodegenerative disease, stroke, head trauma, and possibly emotional disorders such as depression and addiction (Eisch \&

Address for Correspondence: Peter J. Clark, Beckman Institute, 405 N. Mathews Avenue, Urbana, IL 61801, Phone: (217) 333-7123, Fax: (217) 244-5180, pjclark2@illinois.edu. 
Harburg 2006; Kempermann et al. 2008; Leuner et al. 2007; Sahay \& Hen 2007; van Praag et al. 2002).

Artificially replicating the microenvironment in the dentate gyrus responsible for increasing neurogenesis will be extremely difficult. The process involves activation of granule neurons (Deisseroth et al. 2004), extracellular changes in neurochemistry (Bequet et al. 2001; Neeper et al. 1995), changes in gene expression (Neeper et al. 1996), and changes in vascular density (Clark et al. 2009; Van der Borght et al. 2009). An alternative approach is to identify specific molecules or biological pathways that increase sensitivity to a natural stimulator of neurogenesis such as aerobic exercise (van Praag et al. 1999b). Exercise increases the rate of neurogenesis, resulting in increased total numbers of granule neurons and volume of the entire granule cell layer (Clark et al. 2009; Clark et al. 2008; Rhodes et al. 2003). If biological pathways could be manipulated to increase exercise-induced neurogenesis, then it might be possible to accelerate or enhance the integration of new neurons into hippocampal circuits by combining a therapeutic manipulation with an exercise intervention.

One strategy is to identify strains of mice that display extremely high versus extremely low levels of neurogenesis in response to exercise (Rhodes et al. 2003). Such strains can then be contrasted to discover common genetic and neurobiological differences that contribute to the differential sensitivity. Previous studies have characterized strain differences in baseline levels of adult hippocampal neurogenesis when the animals are housed under sedentary conditions (Kempermann et al. 2006; Kempermann \& Gage 2002a, b; Kempermann et al. 1997). However, recent evidence suggests that different genetic pathways may influence baseline sedentary levels of neurogenesis as compared to exercise-induced neurogenesis (Thuret et al. 2009). Moreover, the magnitude of changes in neurogenesis from exercise exceeds natural genetic variation in neurogenesis under sedentary conditions (Rhodes et al. 2003). Hence, an analysis of genetic variation in exercise-induced neurogenesis contributes unique information toward understanding how to effectively grow and incorporate new nerve cells into brain circuits.

Prior to this report, most of what we knew about exercise-induced neurogenesis in mice was conducted in a single mouse strain, C57BL/6J and recently a few others (Bednarczyk et al. 2009; Clark et al. 2009; Rhodes et al. 2003; Thuret et al. 2009). Therefore, the goal of this study was to compare exercise-induced adult hippocampal neurogenesis in 12 different genetically divergent mouse strains. Results identify specific genotypes that display high levels of exercise-induced neurogenesis and other genotypes that display low levels of neurogenesis in response to exercise. Future studies could contrast these mouse strains to identify molecular or neurobiologial factors common to the high responding strains and uncommon in the low strains that enhance the growth of new nerve cells in the adult hippocampus.

\section{Material and Methods}

\section{Animals}

A total of 192 mice from twelve different isogenic strains were used in this study $(\mathrm{n}=16$ per strain): 129S1/SvImJ, AKR/J, B6129SF1/J, B6D2F1/J, BALB/cByJ, BTBR T+ tf/J, C57BL/ 10J, C57BL/6J, CAST/EiJ, DBA/2J, NOD/ShiLtJ, and SM/J. An equal number of males and females $(n=8$ per sex) were represented in each strain, with the exception of BTBR $T+t f / J$ $(\mathrm{n}=9$ females, $\mathrm{n}=7$ males). These strains were chosen for several reasons. First, they are all isogenic, i.e., same sex members of a strain are all genetically identical. This is useful for partitioning phenotypic variation into genetic and environmental components (Crabbe et al. 1990). It is also useful because the genotypes are reproducible, indefinitely. Different investigators over many years can establish information about the specific genotypes used 
herein. Second, the strains are broadly distributed on the phylogeny of laboratory mouse strains, i.e., they are genetically diverse (Rhodes et al. 2007). Third, they are listed as priority in the Mouse Phenome Database. Hence, these stains are among the most widely used in biomedical research and becoming incorporated into a database of genetic and phenotypic information. Fourth, the F1 hybrid strains, B6D2F1/J and B6129SF1/J were included to incorporate heterozygosity in the estimation of genetic differences. The F1 hybrids are isogenic but also heterozygous at all polymorphic loci segregating in the parental strains. B6129SF1/J is the cross between a C57BL/6J female and a 129S1/SvImJ male. $\mathrm{B} 6 \mathrm{D} 2 \mathrm{~F} 1 / \mathrm{J}$ is the cross between a $\mathrm{C} 57 \mathrm{BL} / 6 \mathrm{~J}$ female and a DBA/2J male.

The experiments were conducted in two batches ( $\mathrm{n}=96$ per batch) that consisted of 8 mice per stain equally divided by sex ( $n=4$ males, $n=4$ females), with the exception of BTBR T $+\mathrm{tf} / \mathrm{J}(\mathrm{n}=3$ males, $\mathrm{n}=5$ females in the second batch).

\section{Husbandry}

The two cohorts of mice arrived at the Beckman Institute Animal facility from The Jackson Laboratory (Bar Harbor, ME) at exactly 5 weeks of age. Upon arrival mice were housed four per cage by sex in standard polycarbonate shoebox cages (dimensions $29 \times 19 \times 13 \mathrm{~cm}$ L $\times W \times H$ ) with corncob bedding, $70971 / 4$ (Harlan Teklad, Madison, Wisconsin, USA) for 3 weeks until they were individually housed in cages with or without wheels as described below. Rooms were controlled for temperature $\left(21^{\circ} \mathrm{C}\right)$ and photo-period (12-h L:D; lights on at $7 \mathrm{am}$ and off at $7 \mathrm{pm}$ ). Food (Harlan Teklad 7012) and water were provided ad libitum. The Beckman Institute Animal Facility is AAALAC approved. All procedures were approved by the University of Illinois Institutional Animal Care and Use Committee and adhered to NIH guidelines. All efforts were made to minimize the number of animals used and their suffering.

\section{Experimental Design}

Eight week-old mice were individually housed in cages with (runners) or without (sedentary) running wheels for 43 days. Mice were divided equally by strain and sex into runner and sedentary conditions. Dimensions of running wheel cages were $36 \times 20 \times 14 \mathrm{~cm}$ $(\mathrm{L} \times \mathrm{W} \times \mathrm{H})$ with a $23 \mathrm{~cm}$ diameter wheel mounted in the cage top (Respironics, Bend, OR). Wheel rotations were monitored continuously in $1 \mathrm{~min}$ increments throughout the experiment via magnetic switches interfaced to a computer. The first 10 days all mice received daily injections of 5-Bromo- $2^{\prime}$-deoxyuridine (BrdU; $50 \mathrm{mg} / \mathrm{kg}$ ) to label dividing cells. The first 13 days, sedentary mice were housed in custom made home cages for video tracking. Dimensions of the cages were $18.5 \times 33.5 \times 16 \mathrm{~cm}$, constructed of clear plastic with food and water access mounted on the side. Two different types of bedding were used depending on whether the mouse had a white or a dark coat color. Corncob bedding (Harlan 7097) was used for dark mice whereas Sheppard Paperchip® bedding was used for white mice (see Zombeck et al. 2010a; Zombeck et al. 2010b). Horizontal distance traveled was recorded continuously using TopScan (Clever Sys, Vienna, VA, USA) video tracking software. Following day 13, sedentary mice were transferred to standard polycarbonate shoebox cages (no wheels) where they remained individually housed for 30 more days.

\section{Immunohistochemistry}

Following Clark et al. (2008) animals were anesthetized with $150 \mathrm{mg} / \mathrm{kg}$ sodium pentobarbital (ip) and then perfused transcardially with $4 \%$ paraformaldehyde in a phosphate buffer solution (PBS). Brains were post-fixed overnight, and transferred to $30 \%$ sucrose in PBS. Brains were sectioned using a cryostat into 40 micron coronal sections and stored in tissue cryoprotectant at $-20^{\circ} \mathrm{C}$. Two separate 1-in-6 series of these sections (i.e., series of 
sections throughout the rostro-caudal extent of the brain with 240 micron increments separating each section) were stained in each of the following ways.

1. BrdU-DAB. Purpose: To detect the number of BrdU-positive newly divided cells, located adjacent to or in the granule cell layer. Free floating sections were washed in tris-buffering solution (TBS) and then treated with $0.6 \%$ hydrogen peroxide. To denature DNA for BrdU detection, sections were pre-treated with $50 \%$ de-ionized formamide, $10 \%$ 20XSCC buffer, 2N hydrochloric acid, $0.1 \mathrm{M}$ Boric acid. Sections were blocked with a solution of $0.3 \%$ Triton-X and 3\% goat serum in TBS (TBS-X plus), and then incubated in primary antibody against BrdU made in rat (Accurate, Westbury, NY) at a dilution of 1:100 in TBS-X plus for $72 \mathrm{hrs}$ at $4 \mathrm{oC}$. Sections were then washed in TBS, treated with TBS-X plus for $30 \mathrm{~min}$ and then incubated in secondary antibody against rat made in goat at 1:250 in TBS-X plus for $100 \mathrm{~min}$ at room temperature. Sections were then treated using the ABC system (Vector, Burlingame, CA) and stained using a diaminobenzidine kit (Sigma, St. Louis, MO).

2. Double fluorescent label. Purpose: To determine the proportion of BrdU-positive cells $(\mathrm{BrdU}+)$ in the dentate gyrus that differentiated into neurons. The same procedure as 1 was used except a cocktail was used for the primary antibody step, rat anti-BrdU (1:100; Accurate, Westbury, NY) and mouse anti-NeuN (1:50; Chemicon, Billerica, MA), together. Secondary antibodies made in goat were conjugated with fluorescent markers (Cy3 anti-rat, $\mathrm{Cy} 2$-anti mouse) at dilution 1:200 and also delivered as a cocktail.

\section{Image analysis}

1. BrdU-DAB. Following Clark et al. (2008), the entire granule layer (bilateral), represented in the 1-in-6 series was photographed by systematically advancing the field of view of the Zeiss brightfield light microscope, and taking multiple photographs, via camera interfaced to computer, under 10X (total 100X) magnification. Positively labeled cells in these photographs were automatically counted using ImageJ software. We validated the automation by measuring 176 sections using both the automated method and manually (i.e., counting the nuclei by eye under the light microscope). The Pearson's correlation was 0.99 and the linear regression was not different from the 1-to-1 line. To obtain unbiased estimates of BrdU cell numbers, total counts were multiplied by 0.85 , under the assumption that $15 \%$ of the nuclei counted would intersect with the plane of the section. This was estimated based on the observation that the average size of BrdU nuclei was 6 microns, which is $15 \%$ of 40 microns, the thickness of the section. In addition, the total volume of the dentate gyrus represented in the series was measured so that the counts could be expressed per cubic micrometer dentate gyrus sampled.

2. Double label. A confocal Leica SP2 laser scanning confocal microscope (using a 40X oil objective, pinhole size $81.35 \mu \mathrm{m}$ ) was used to determine the proportion of BrdU positive cells differentiated into neurons ( $\mathrm{NeuN}+$ ). Each BrdU positive cell in the granular layer (represented in the 1-in- 6 series) was analyzed by focusing through the tissue in the z-axis to establish co-labeling with NeuN. The number of new neurons per cubic micrometer per mouse was calculated as the number of BrdU cells per cubic micrometer multiplied by average proportion BrdU cells coexpressing NeuN. 


\section{Statistical Analysis}

Data were analyzed using SAS or R statistical software. $P<0.05$ was considered statistically significant. Home cage activity (average distance traveled in cages without wheels) and wheel running (average distance run) were analyzed by 2-way ANOVA with strain (12 genotypes), sex (male versus female) and the interaction between strain and sex as factors.

Adult hippocampal neurogenesis was measured as the total number of BrdU positive cells multiplied by the estimated fraction of BrdU cells expressing NeuN per volume granule layer. Adult neurogenesis and volume of the granule cell layer were analyzed by 3-way ANOVA with strain (12 genotypes), sex (male versus female), treatment (sedentary versus runner) and all interactions entered in the model. Adult hippocampal neurogenesis was also analyzed separately for sedentary mice and runners using analysis of covariance (ANCOVA) with strain entered as the categorical variable and average distance traveled in cages without wheels (by video tracking) or average distance traveled on running wheels entered as the continuous covariates, respectively. Tukey tests were used to evaluate posthoc comparisons of means between groups.

The proportion of BrdU labeled cells in the granule cell layer that also expressed NeuN was analyzed by logistic regression. For these analyses, the deviance is reported in place of an F statistic.

Phenotypic correlations were estimated as Pearson's $r$ between individual trait values. Genetic correlations were estimated as Pearson's $r$ and Spearman's $\rho$ between strain means for two different traits. Outlier strains for genetic correlations were determined using a linear model that predicts one trait (strain mean) as a function of another plus a categorical variable that differentiates the outlier-in-question from the rest of the data-points.

\section{Results}

\section{Sedentary levels of physical activity}

Strains significantly varied with respect to average distance traveled in home cages without wheels (see Fig. 1A). On average, males moved $88 \%$ as far as females across all strains. This was reflected in a significant effect of strain $\left(\mathrm{F}_{11,72}=12.2, P<0.0001\right)$ and sex $\left(\mathrm{F}_{1,72}=4.2, P=0.045\right)$, but not the interaction between strain and sex $(P>0.05)$. Cast/EiJ moved the most $(0.49 \mathrm{~km} / \mathrm{day} \pm 0.066 \mathrm{SE}$, collapsed across sex $)$ whereas $129 \mathrm{~S} 1 / \mathrm{SvImJ}$ moved the least $(0.13 \mathrm{~km} /$ day $\pm 0.016 \mathrm{SE})$.

\section{Wheel running}

Strains significantly varied with respect to average distance traveled on running wheels (see Fig. 1B). On average, males ran $80 \%$ as far as females across all strains. This was reflected by a significant effect of $\operatorname{strain}\left(\mathrm{F}_{11,72}=8.1, P<0.0001\right)$ and $\operatorname{sex}\left(\mathrm{F}_{1,72}=10.7, P=0.002\right)$, but not the interaction between strain and sex. B6129SF1/J ran the most $(10.2 \mathrm{~km} /$ day \pm 0.88 $\mathrm{SE}$, collapsed across sex) whereas $129 \mathrm{~S} 1 / \mathrm{SvImJ}$ ran the least $(2.8 \mathrm{~km} / \mathrm{day} \pm 0.79 \mathrm{SE}$, collapsed across sex).

\section{Body mass}

Wheel running exercise significantly reduced body mass across all strains and sexes. This was reflected by a significant main effect of treatment $\left(\mathrm{F}_{1,138}=5.8, \mathrm{P}=0.02\right)$, strain $\left(\mathrm{F}_{11,138}=55.1, \mathrm{P}<0.0001\right)$ and $\operatorname{sex}\left(\mathrm{F}_{1,138}=167.5, \mathrm{P}<0.0001\right)$ but no interactions. On average, runners were $97 \%$ as heavy as sedentary animals, and females were $84 \%$ as heavy as males. CAST/EiJ mice were the smallest $(12.9 \mathrm{~g} \pm 0.29 \mathrm{SE}$, collapsed across sex and exercise conditions) whereas BTBR $\mathrm{T}+\mathrm{tf} / \mathrm{J}$ were the largest $(29.8 \mathrm{~g} \pm 0.87 \mathrm{SE})$. 


\section{Adult hippocampal neurogenesis}

In this study, neurogenesis is defined as the total number of BrdU positive cells per volume granule cell layer of the dentate gyrus multiplied by the estimated fraction of $\mathrm{BrdU}$ positive cells differentiated into neurons (i.e., co-labeled with NeuN, see Neuronal differentiation below). Neurogenesis represented per volume was highly correlated with the total number of BrdU positive neurons (not corrected by volume) across all subjects ( $\mathrm{n}=192$, Pearson's $r=0.94, \mathrm{P}<0.0001)$. This is because the coefficient of variation (CV) for the total number of BrdU neurons was much larger than for estimated volumes (CV was 0.83 versus 0.21 , respectively), hence dividing by volume had only a small effect on changing relative differences in total BrdU neuron numbers. Total numbers of BrdU neurons are reported in Table 1 and were significantly correlated with volume of the granule layer across all subjects ( $\mathrm{n}=192$, Pearson's $r=0.47, \mathrm{P}<0.0001$ ).

Sedentary density of new neurons-Baseline density of new neurons in sedentary mice significantly varied depending on genotype (Fig. 2). This was reflected by a significant effect of strain $\left(\mathrm{F}_{11,72}=8.1, P<0.0001\right)$. The effect of sex and the interaction between sex and strain were not significant (both $P>0.05$ ). C57BL/6J mice showed the highest amount of neurogenesis under sedentary conditions $\left(6.3 \times 10^{3}\right.$ new neurons per cubic $\left.\mathrm{mm} \pm 0.58 \mathrm{SE}\right)$ whereas DBA/2J displayed the lowest $\left(2.0 \times 10^{3}\right.$ new neurons per cubic $\left.\mathrm{mm} \pm 0.27 \mathrm{SE}\right)$.

Exercise-induced neurogenesis-Exercise significantly increased adult hippocampal neurogenesis in all the strains. However, the magnitude of the effect depended on genotype (Fig. 2). This was reflected by a significant effect of strain $\left(\mathrm{F}_{11,144}=5.2, P<0.0001\right)$, treatment (runner versus sedentary) $\left(\mathrm{F}_{1,144}=349.1, P<0.0001\right)$ and the interaction between strain and treatment $\left(\mathrm{F}_{11,144}=3.3, P=0.0005\right)$. All pairwise Tukey tests comparing sedentary versus runners within each strain were significant (all $P<0.05$ ). The main effect of sex and all interactions between sex and the other variables were not significant (all $\mathrm{P}<0.05$ ).

C57BL/6J was the least responsive strain. Running increased neurogenesis by only 1.6 fold whereas all other strains showed more than a doubling in neurogenesis from running. The most responsive strains increased neurogenesis by $4-5$ fold, including AKR/J, B6129SF1/J, $\mathrm{BALB} / \mathrm{cByJ}, \mathrm{CAST} / \mathrm{EiJ}$ and SM/J.

Volume of the granule cell layer of the dentate gyrus-Strains significantly differed for volume of the dentate gyrus (Table 1). Wheel running increased volume across all strains by a similar magnitude (approximately $9 \%$ see below). This was reflected by a significant effect of strain $\left(\mathrm{F}_{11,144}=10.6, P<0.0001\right)$ and treatment $\left(\mathrm{F}_{1,144}=15.4, P<0.0001\right)$ in the 3-way ANOVA but no significant effects of sex or any interactions. On average across all animals, the granule cell layer in sedentary mice was $91 \%$ the volume of runners. Collapsed across treatment, BTBR $\mathrm{T}+\mathrm{tf} / \mathrm{J}$ displayed the smallest volume at 0.34 cubic $\mathrm{mm}$ $( \pm 0.014 \mathrm{SE})$ whereas B6129SF1/J had the largest at 0.52 cubic $\mathrm{mm}( \pm 0.026 \mathrm{SE})$.

Neuronal differentiation-We analyzed a total of 4,835 BrdU positive cells (age 34-43 days old) in the granule layer of the dentate gyrus by confocal microscopy to determine whether they also expressed the neuronal marker NeuN. An average of $61 \mathrm{BrdU}$ positive cells per strain and sex were analyzed for sedentary animals and 140 per strain and sex for runners. Running increased the percentage of BrdU cells displaying NeuN by a similar amount in all strains and sexes (see Table 1). This was reflected by a significant main effect of treatment (runner versus sedentary) (Deviance $=6.2, P<0.0001$ ) in the logistic regression, but no significant main effects of strain, sex or any interactions (all $P>0.05$ ). Collapsed across strain and sex, the average percentage of BrdU cells displaying NeuN in sedentary mice was $78 \%( \pm 1.1)$ and for runners it was $90 \%( \pm 0.5)$. 


\section{Analysis of covariance}

Home cage activity as the covariate-Individual distance traveled in the home cages of sedentary animals (without running wheels) was not significantly correlated with levels of neurogenesis across animals (Pearson's $r$ was $0.05, P>0.05$ ) (Fig. 3A). In the analysis of covariance, strain was significant $\left(\mathrm{F}_{11,72}=8.5, P<0.0001\right)$, but distance traveled and the interaction between distance and strain were not $(\mathrm{P}>0.05)$.

Wheel running as the covariate-Individual distance run was significantly correlated with density of new neurons across all animals (Pearson's $r$ was $0.49, P<0.0001$ ). However, certain strains displayed significantly more new neurons than other strains for a given level of wheel running behavior (Fig. 3B). This was reflected by a significant effect of distance run $\left(\mathrm{F}_{1,72}=33.8, P<0.0001\right)$ and strain $\left(\mathrm{F}_{11,72}=2.6, P=0.008\right)$ but no interaction $(P>0.05)$ in the analysis of covariance, where individual distance run was entered as a continuous variable and strain as a categorical variable.

\section{Genetic correlations}

Between physical activity traits-Wheel running behavior and levels of physical activity in home cages without wheels (as measured by video tracking) were not significantly genetically correlated. This was reflected by a non-significant strain mean correlation between distance run on wheels and distance traveled in home cages without wheels ( $\mathrm{n}=12$, Pearson's $r=0.46$, Spearman's $\rho=0.46, P>0.05$ ) (see Fig. 4A). However, B6129SF1/J was a significant outlier $\left(\mathrm{F}_{1,9}=17.1, P=0.003\right)$. It displayed relatively low levels of activity without wheels (approximately $0.2 \mathrm{~km} /$ day) but the highest levels of wheel running (approximately $10 \mathrm{~km} /$ day). Without B6129SF1/J, the genetic correlation was significant (n=11, Pearson's $r=0.74, P=0.009$; Spearman's $\rho=0.73, P=0.02$ ).

Between physical activity and neurogenesis-Physical activity was not significantly genetically correlated with levels of neurogenesis under sedentary conditions (Fig. 4B). Pearson's $r$ for the strain-mean correlation between distance traveled in cages without wheels and number of new neurons was -0.08 ( $\mathrm{n}=12, P>0.05$; Spearman's $\rho=0.15, P>0.05$ ). However, a significant genetic correlation was observed between wheel running and adult hippocampal neurogenesis (Fig. 4C). Pearson's $r$ for the strain-mean correlation between distance run on wheels and number of new neurons was 0.66 ( $\mathrm{n}=12, P=0.02$; Spearman's $\rho=$ $0.62, P=0.03)$.

Between neurogenesis in sedentary and runner conditions-Neurogenesis measured in standard sedentary conditions was not significantly genetically correlated with neurogenesis measured in animals housed with running wheels (Fig. 4D). Pearson's $r$ for the strain-mean correlation between neurogenesis in runners versus sedentary animals was 0.30 $(\mathrm{n}=12, P>0.05$; Spearman's $\rho=0.29, P>0.05)$.

\section{Discussion}

Our results illustrate the robustness of the effects of wheel running on increasing adult hippocampal neurogenesis in mice and the importance of background genetics for determining the magnitude of the neuroplastic response. The significant gene by environment interaction for the effects of running on neurogenesis across strains implies that it should be possible to find plasticity genes or genes that predispose large versus small enhancement in neurogenesis from running. The list of reasonable candidates from the literature is large and includes, but is not limited to, genes that regulate signaling from trophic factors such as brain derived neurotrophic factor (BDNF), nerve growth factor (NGF), neurotrophin-3 and growth factors such as insulin like growth factor 1 (IGF-1), 
fibroblast growth factor 2 (FGF-2), and vascular endothelial growth factor (VEGF), genes that are expressed during neuron development (e.g., Nestin, sox2, NeuroD, TLX, Cdk5, doublecortin, Tis21), and genes that regulate cellular metabolism (e.g., leptin signaling, glucose transport) (Aberg et al. 2000; Fabel et al. 2003; Favaro et al. 2009; Frielingsdorf et al. 2007; Fukuda et al. 2003; Garza et al. 2008; Johnson et al. 2003; Lagace et al. 2008; Membrez et al. 2006; Palmer et al. 1995; Rossi et al. 2006; Seki 2002; Zhang et al. 2008).

An important discovery is that $\mathrm{C} 57 \mathrm{BL} / 6 \mathrm{~J}$, the most frequent mouse strain previously used to characterize effects of wheel running on adult hippocampal neurogenesis, was the least responsive strain to having a running wheel. Running increased neurogenesis in C57BL/6J by 1.6 fold, a level consistent with other reports (Clark et al. 2009; Clark et al. 2008; Clark et al. 2010; van Praag et al. 1999a; van Praag et al. 1999b), whereas all other strains displayed at least a doubling of neurogenesis, and several displayed 4-5 fold increases (see Fig. 2). The low fold response in C57BL/6J relative to other strains was in part due to a relatively high baseline level of neurogenesis and in part to a low level of running. The high baseline level of neurogenesis under sedentary conditions (Fig. 2) is consistent with previous reports (Kempermann et al. 2006; Kempermann \& Gage 2002a, b; Kempermann et al. 1997). Moreover, consistent with literature, C57BL/6J also ran less than other strains (Fig 1B) (Lightfoot et al. 2004) and average daily distance run was significantly correlated with neurogenesis (Fig. 3B, 5C). In the analysis of covariance, C57BL/6J displayed average levels of neurogenesis relative to other strains for a fixed level of running. Hence, the C57BL/6J genotype may not be the ideal model organism to study exercise-induced neurogenesis because the change in neurogenesis from running is relatively small in comparison to other strains, and therefore, statistical power to find correlated responses is limited.

Another important discovery was that genetic variation in neurogenesis under standard housing conditions was unrelated to running levels of neurogenesis (Fig 4D). The lack of genetic correlation suggests that different genes influence variation in adult hippocampal neurogenesis under sedentary versus runner conditions. Previous work has characterized genetic variation in levels of adult hippocampal neurogenesis among laboratory strains of mice under standard conditions similar to our sedentary treatment, without running wheels or enrichment (Kempermann \& Gage 2002a,b;Kempermann et al. 1997). Recent work has begun mapping genes that influence variation in levels of neurogenesis under these standard sedentary conditions in genetic reference populations (e.g, BXD, AXB) (Kempermann et al. 2006; Philip et al. 2010). Our findings suggest that the genes and pathways discovered in these studies will likely be different from those that increase neurogenesis in response to running. If the goal is to identify genes and biological pathways that enhance neurogenesis, it will be useful to include aerobic exercise in the analysis because results demonstrate that the greatest increases in neurogenesis are produced from combining the appropriate genes with the appropriate environmental conditions.

To the best of our knowledge, this is the first study to examine the relationship between physical activity in the home cage without a running wheel and numbers of new neurons in the hippocampus. Strains differed in how physically active they were in cages without wheels (Fig. 1A). However, physical activity was not correlated with adult hippocampal neurogenesis in animals housed in cages without wheels (Fig. 3A). This contrasts markedly with results for animals housed with running wheels. Individual level of running was significantly correlated with adult hippocampal neurogenesis (Fig. 3B), a result that has been reported before for individuals within strains (Allen et al. 2001;Bednarczyk et al. 2009; Clark et al. 2009; Rhodes et al. 2003). We interpret this finding to imply that physical activity must reach a certain threshold level that taxes aerobic capacity before it can increase 
neurogenesis in a quantitative fashion (Chaddock et al. 2010;Erickson et al. 2009; Pereira et al. 2007).

Adult hippocampal neurogenesis is multi-stage process that occurs over a period of weeks in rodents (van Praag et al. 2002). It requires stem cell proliferation, survival of daughter cells, differentiation into neurons, and integration into circuits. In this study we measured net neurogenesis resulting from the entire process occurring over six weeks because in the end, the survival and integration of new neurons is what matters for regenerative medicine. We did not attempt to separate the contribution of proliferation versus survival as was done in early studies (e.g., Kempermann \& Gage 2002a, b; van Praag et al. 1999b). Theoretically, exercise could increase neurogenesis by increasing proliferation, survival or differentiation. Although early reports suggested that wheel running increases neurogenesis primarily by increasing proliferation (Olson et al. 2006; van Praag et al. 1999b), more recent studies in C57BL/6J show that the effect of increased proliferation from wheel running is small and transient and that the major factor sustaining increased neurogenesis from wheel running is increased survival and differentiation (Clark et al.; Fuss et al. 2009; Kronenberg et al. 2006; Snyder et al. 2009). Future work is needed to determine whether survival and differentiation are the major factors contributing to increased neurogenesis from exercise in the other genotypes.

The strains of mice compared in our study were all isogenic, meaning that same sex individuals within a strain were genetically identical. We used this approach to separate genetic from environmental contributions to phenotypic variation. When individuals from a strain are all genetically identical, variation within strains can only be attributed to environment whereas variation between strains can be attributed to genetic influences, assuming contributions of maternal effects and early rearing environment to between-strain variation are minimal (Crabbe et al. 1990; Rhodes et al. 2007). Using this strategy, we found evidence for significant genetic influences on physical activity, neurogenesis, and volume of the granule layer of the dentate gyrus. Broad sense heritability estimates, representing $R^{2}$ values from 1-way ANOVAs with strain as the factor, are summarized in Table 2. The results suggest that it should be possible to find genes or specific locations in the DNA where changes occur between strains that explain the heritable variation in the phenotypes (Kempermann et al. 2006).

An interesting finding was that the pattern of inheritance for physical activity and neurogenesis in the F1 hybrids was different under sedentary and runner conditions. Wheel running behavior and running-induced neurogenesis showed significant over-dominance or heterosis. The hybrids ran more (Fig. 1B) and grew more new neurons when exposed to running wheels (Fig. 2C) as compared to either of their parental strains (all posthoc $P<0.05$ ). This result suggests that heterozygosity (having alternative alleles at a single locus) increases exercise-induced adult hippocampal neurogenesis above and beyond the additive contributions of each allele as measured separately in the homozygous state. The implication is that combinations of alternative gene transcripts or expression patterns produce the greatest increases in wheel running and exercise-induced neurogenesis. In contrast, home cage activity (Fig. 1A) and sedentary levels of neurogenesis (Fig. 2C) showed additive or dominant effects. The F1 hybrids were intermediate to the parental strains, or not significantly different from C57BL/6J.

We identified 2 low and 4 high responding strains that could be contrasted in a future research program to discover genes and biological correlates of enhanced or suppressed adult hippocampal neurogenesis. The following 2 strains displayed low levels of exerciseinduced neurogenesis relative to the other strains and low levels for a fixed amount of running as indicated by analysis of covariance: $129 \mathrm{~S} 1 / \mathrm{SvImJ}$ and DBA/2J. The following 4 
strains displayed high levels neurogenesis for a fixed amount of running and high levels overall: AKR/J, C57BL/10J, BALB/cByJ, and NOD/ShiLtJ. These particular strains deserve follow up examination for correlated features at multiple levels of biological organization from molecules to behavior. For example, it would be useful to find changes in gene expression in the hippocampus from running that are common in the high responding strains and uncommon in the low responding strains. Such genes would represent candidates for increasing sensitivity to exercise-induced neurogenesis that could be directly tested using knockin or knockdown approaches such as transgenic mice carrying multiple copies of the gene or RNA interference, respectively. Moreover, if the same strains were tested for effects of exercise on learning and memory tasks, it would be possible to determine whether the strains that display the greatest levels of exercise-induced adult hippocmapal neurogenesis also display the greatest enhancement in behavioral performance from exercise. Such data would bring useful information to the debate about the functional significance of exercise induced neurogenesis in behavioral performance (Kempermann 2002; Schinder \& Gage 2004; van Praag 2009). Analysis of exercise-induced neurogenesis in more strains including BXD Recombinant Inbred lines would substantially increase the power for testing these and other related hypotheses (Kempermann et al. 2006).

Artificially replicating the microenvironment in the brain responsible for increasing neurogenesis for therapeutics may be difficult. But, it may be possible to identify genes or specific molecules that increase sensitivity to a natural stimulator of plasticity in the adult mammalian brain, aerobic physical activity. Finding genes and biological pathways that differentiate high and low responders represents a promising approach. In the future, it might be possible to manipulate a specific pathway in the brain that in combination with exercise could enhance the growth and incorporation of new nerve cells into the adult hippocampus.

\section{Acknowledgments}

This work was supported by grants from NIH, MH083807 and DA027487 to JSR.

\section{References}

Aberg MA, Aberg ND, Hedbacker H, Oscarsson J, Eriksson PS. Peripheral infusion of IGF-I selectively induces neurogenesis in the adult rat hippocampus. J Neurosci. 2000; 20:2896-2903. [PubMed: 10751442]

Allen DM, van Praag H, Ray J, Weaver Z, Winrow CJ, Carter TA, Braquet R, Harrington E, Ried T, Brown KD, Gage FH, Barlow C. Ataxia telangiectasia mutated is essential during adult neurogenesis. Genes Dev. 2001; 15:554-566. [PubMed: 11238376]

Bednarczyk MR, Aumont A, Decary S, Bergeron R, Fernandes KJ. Prolonged voluntary wheelrunning stimulates neural precursors in the hippocampus and forebrain of adult CD1 mice. Hippocampus. 2009; 19:913-927. [PubMed: 19405143]

Bequet F, Gomez-Merino D, Berthelot M, Guezennec CY. Exercise-induced changes in brain glucose and serotonin revealed by microdialysis in rat hippocampus: effect of glucose supplementation. Acta Physiol Scand. 2001; 173:223-230. [PubMed: 11683680]

Chaddock L, Erickson KI, Prakash RS, Kim JS, Voss MW, Vanpatter M, Pontifex MB, Raine LB, Konkel A, Hillman CH, Cohen NJ, Kramer AF. A neuroimaging investigation of the association between aerobic fitness, hippocampal volume and memory performance in preadolescent children. Brain Res. 2010 In press.

Clark PJ, Brzezinska WJ, Puchalski EK, Krone DA, Rhodes JS. Functional analysis of neurovascular adaptations to exercise in the dentate gyrus of young adult mice associated with cognitive gain. Hippocampus. 2009; 19:937-950. [PubMed: 19132736] 
Clark PJ, Brzezinska WJ, Thomas MW, Ryzhenko NA, Toshkov SA, Rhodes JS. Intact neurogenesis is required for benefits of exercise on spatial memory but not motor performance or contextual fear conditioning in C57BL/6J mice. Neuroscience. 2008; 155:1048-1058. [PubMed: 18664375]

Clark PJ, Kohman RA, Miller DS, Bhattacharya TK, Haferkamp EH, Rhodes JS. Adult hippocampal neurogenesis and c-Fos induction during escalation of voluntary wheel running in C57BL/6J mice. Behav Brain Res. 2010; 213:246-252. [PubMed: 20472002]

Crabbe JC, Phillips TJ, Kosobud A, Belknap JK. Estimation of genetic correlation: interpretation of experiments using selectively bred and inbred animals. Alcohol Clin Exp Res. 1990; 14:141-151. [PubMed: 2190477]

Deisseroth K, Singla S, Toda H, Monje M, Palmer TD, Malenka RC. Excitation-neurogenesis coupling in adult neural stem/progenitor cells. Neuron. 2004; 42:535-552. [PubMed: 15157417]

Deng W, Aimone JB, Gage FH. New neurons and new memories: how does adult hippocampal neurogenesis affect learning and memory? Nat Rev Neurosci. 2010; 11:339-350. [PubMed: 20354534]

Eisch AJ, Harburg GC. Opiates, psychostimulants, and adult hippocampal neurogenesis: Insights for addiction and stem cell biology. Hippocampus. 2006; 16:271-286. [PubMed: 16411230]

Erickson KI, Prakash RS, Voss MW, Chaddock L, Hu L, Morris KS, White SM, Wojcicki TR, McAuley E, Kramer AF. Aerobic fitness is associated with hippocampal volume in elderly humans. Hippocampus. 2009; 19:1030-1039. [PubMed: 19123237]

Fabel K, Tam B, Kaufer D, Baiker A, Simmons N, Kuo CJ, Palmer TD. VEGF is necessary for exercise-induced adult hippocampal neurogenesis. Eur J Neurosci. 2003; 18:2803-2812. [PubMed: 14656329]

Favaro R, Valotta M, Ferri AL, Latorre E, Mariani J, Giachino C, Lancini C, Tosetti V, Ottolenghi S, Taylor V, Nicolis SK. Hippocampal development and neural stem cell maintenance require Sox2dependent regulation of Shh. Nat Neurosci. 2009; 12:1248-1256. [PubMed: 19734891]

Frielingsdorf H, Simpson DR, Thal LJ, Pizzo DP. Nerve growth factor promotes survival of new neurons in the adult hippocampus. Neurobiol Dis. 2007; 26:47-55. [PubMed: 17270453]

Fukuda S, Kato F, Tozuka Y, Yamaguchi M, Miyamoto Y, Hisatsune T. Two distinct subpopulations of nestin-positive cells in adult mouse dentate gyrus. J Neurosci. 2003; 23:9357-9366. [PubMed: 14561863]

Fuss J, Ben Abdallah NM, Vogt MA, Touma C, Pacifici PG, Palme R, Witzemann V, Hellweg R, Gass $\mathrm{P}$. Voluntary exercise induces anxiety-like behavior in adult C57BL/6J mice correlating with hippocampal neurogenesis. Hippocampus. 2009

Garza JC, Guo M, Zhang W, Lu XY. Leptin increases adult hippocampal neurogenesis in vivo and in vitro. J Biol Chem. 2008; 283:18238-18247. [PubMed: 18367451]

Johnson RA, Rhodes JS, Jeffrey SL, Garland T Jr, Mitchell GS. Hippocampal brain-derived neurotrophic factor but not neurotrophin-3 increases more in mice selected for increased voluntary wheel running. Neuroscience. 2003; 121:1-7. [PubMed: 12946694]

Kempermann G. Why new neurons? Possible functions for adult hippocampal neurogenesis. J Neurosci. 2002; 22:635-638. [PubMed: 11826092]

Kempermann G, Chesler EJ, Lu L, Williams RW, Gage FH. Natural variation and genetic covariance in adult hippocampal neurogenesis. Proc Natl Acad Sci U S A. 2006; 103:780-785. [PubMed: 16407118]

Kempermann G, Gage FH. Genetic determinants of adult hippocampal neurogenesis correlate with acquisition, but not probe trial performance, in the water maze task. Eur J Neurosci. 2002a; 16:129-136. [PubMed: 12153537]

Kempermann G, Gage FH. Genetic influence on phenotypic differentiation in adult hippocampal neurogenesis. Brain Res Dev Brain Res. 2002b; 134:1-12.

Kempermann G, Krebs J, Fabel K. The contribution of failing adult hippocampal neurogenesis to psychiatric disorders. Curr Opin Psychiatry. 2008; 21:290-295. [PubMed: 18382230]

Kempermann G, Kuhn HG, Gage FH. Genetic influence on neurogenesis in the dentate gyrus of adult mice. Proc Natl Acad Sci U S A. 1997; 94:10409-10414. [PubMed: 9294224] 
Kronenberg G, Bick-Sander A, Bunk E, Wolf C, Ehninger D, Kempermann G. Physical exercise prevents age-related decline in precursor cell activity in the mouse dentate gyrus. Neurobiol Aging. 2006; 27:1505-1513. [PubMed: 16271278]

Lagace DC, Benavides DR, Kansy JW, Mapelli M, Greengard P, Bibb JA, Eisch AJ. Cdk5 is essential for adult hippocampal neurogenesis. Proc Natl Acad Sci U S A. 2008; 105:18567-18571. [PubMed: 19017796]

Leuner B, Kozorovitskiy Y, Gross CG, Gould E. Diminished adult neurogenesis in the marmoset brain precedes old age. Proc Natl Acad Sci U S A. 2007; 104:17169-17173. [PubMed: 17940008]

Lightfoot JT, Turner MJ, Daves M, Vordermark A, Kleeberger SR. Genetic influence on daily wheel running activity level. Physiol Genomics. 2004; 19:270-276. [PubMed: 15383638]

Membrez M, Hummler E, Beermann F, Haefliger JA, Savioz R, Pedrazzini T, Thorens B. GLUT8 is dispensable for embryonic development but influences hippocampal neurogenesis and heart function. Mol Cell Biol. 2006; 26:4268-4276. [PubMed: 16705176]

Neeper SA, Gomez-Pinilla F, Choi J, Cotman C. Exercise and brain neurotrophins. Nature. 1995; 373:109. [PubMed: 7816089]

Neeper SA, Gomez-Pinilla F, Choi J, Cotman CW. Physical activity increases mRNA for brainderived neurotrophic factor and nerve growth factor in rat brain. Brain Res. 1996; 726:49-56. [PubMed: 8836544]

Olson AK, Eadie BD, Ernst C, Christie BR. Environmental enrichment and voluntary exercise massively increase neurogenesis in the adult hippocampus via dissociable pathways. Hippocampus. 2006; 16:250-260. [PubMed: 16411242]

Palmer TD, Ray J, Gage FH. FGF-2-responsive neuronal progenitors reside in proliferative and quiescent regions of the adult rodent brain. Mol Cell Neurosci. 1995; 6:474-486. [PubMed: 8581317]

Pereira AC, Huddleston DE, Brickman AM, Sosunov AA, Hen R, McKhann GM, Sloan R, Gage FH, Brown TR, Small SA. An in vivo correlate of exercise-induced neurogenesis in the adult dentate gyrus. Proc Natl Acad Sci U S A. 2007; 104:5638-5643. [PubMed: 17374720]

Philip VM, Duvvuru S, Gomero B, Ansah TA, Blaha CD, Cook MN, Hamre KM, Lariviere WR, Matthews DB, Mittleman G, Goldowitz D, Chesler EJ. High-throughput behavioral phenotyping in the expanded panel of BXD recombinant inbred strains. Genes Brain Behav. 2010; 9:129-159. [PubMed: 19958391]

Rhodes JS, Ford MM, Yu CH, Brown LL, Finn DA, Garland T Jr, Crabbe JC. Mouse inbred strain differences in ethanol drinking to intoxication. Genes Brain Behav. 2007; 6:1-18. [PubMed: 17233637]

Rhodes JS, van Praag H, Jeffrey S, Girard I, Mitchell GS, Garland T Jr, Gage FH. Exercise increases hippocampal neurogenesis to high levels but does not improve spatial learning in mice bred for increased voluntary wheel running. Behav Neurosci. 2003; 117:1006-1016. [PubMed: 14570550]

Rossi C, Angelucci A, Costantin L, Braschi C, Mazzantini M, Babbini F, Fabbri ME, Tessarollo L, Maffei L, Berardi N, Caleo M. Brain-derived neurotrophic factor (BDNF) is required for the enhancement of hippocampal neurogenesis following environmental enrichment. Eur J Neurosci. 2006; 24:1850-1856. [PubMed: 17040481]

Sahay A, Hen R. Adult hippocampal neurogenesis in depression. Nat Neurosci. 2007; 10:1110-1115. [PubMed: 17726477]

Schinder AF, Gage FH. A hypothesis about the role of adult neurogenesis in hippocampal function. Physiology (Bethesda). 2004; 19:253-261. [PubMed: 15381753]

Schmidt-Hieber C, Jonas P, Bischofberger J. Enhanced synaptic plasticity in newly generated granule cells of the adult hippocampus. Nature. 2004; 429:184-187. [PubMed: 15107864]

Seki T. Expression patterns of immature neuronal markers PSA-NCAM, CRMP-4 and NeuroD in the hippocampus of young adult and aged rodents. J Neurosci Res. 2002; 70:327-334. [PubMed: 12391592]

Snyder JS, Glover LR, Sanzone KM, Kamhi JF, Cameron HA. The effects of exercise and stress on the survival and maturation of adult-generated granule cells. Hippocampus. 2009; 19:898-906. [PubMed: 19156854] 
Thuret S, Toni N, Aigner S, Yeo GW, Gage FH. Hippocampus-dependent learning is associated with adult neurogenesis in MRL/MpJ mice. Hippocampus. 2009; 19:658-669. [PubMed: 19140178]

Van der Borght K, Kobor-Nyakas DE, Klauke K, Eggen BJ, Nyakas C, Van der Zee EA, Meerlo P. Physical exercise leads to rapid adaptations in hippocampal vasculature: temporal dynamics and relationship to cell proliferation and neurogenesis. Hippocampus. 2009; 19:928-936. [PubMed: 19212941]

van Praag H. Exercise and the brain: something to chew on. Trends Neurosci. 2009; 32:283-290. [PubMed: 19349082]

van Praag H, Christie BR, Sejnowski TJ, Gage FH. Running enhances neurogenesis, learning, and long-term potentiation in mice. Proc Natl Acad Sci U S A. 1999a; 96:13427-13431. [PubMed: 10557337]

van Praag H, Kempermann G, Gage FH. Running increases cell proliferation and neurogenesis in the adult mouse dentate gyrus. Nat Neurosci. 1999b; 2:266-270. [PubMed: 10195220]

van Praag H, Schinder AF, Christie BR, Toni N, Palmer TD, Gage FH. Functional neurogenesis in the adult hippocampus. Nature. 2002; 415:1030-1034. [PubMed: 11875571]

Zhang CL, Zou Y, He W, Gage FH, Evans RM. A role for adult TLX-positive neural stem cells in learning and behaviour. Nature. 2008; 451:1004-1007. [PubMed: 18235445]

Zombeck JA, DeYoung E, Brzezinska WJ, Rhodes JS. Selective breeding for increased home cage physical activity in Collaborative Cross and Hsd:ICR mice. Behav Genet. 2010a Under review.

Zombeck JA, Swearingen SP, Rhodes JS. Acute locomotor responses to cocaine in adolescents vs. adults from four divergent inbred mouse strains. Genes Brain Behav. 2010b; 9:892-898. [PubMed: 20662938] 

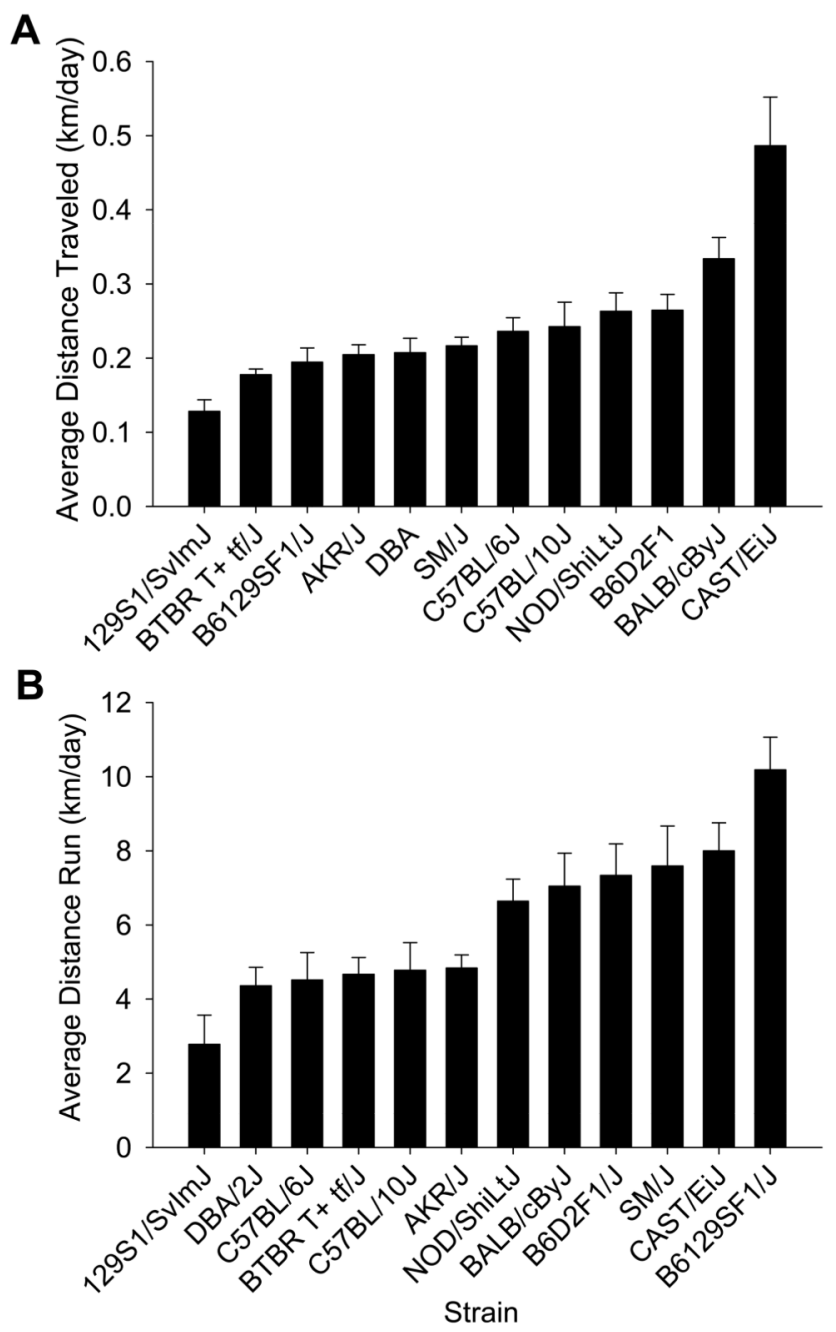

Figure 1.

Genetic differences in home cage activity and wheel running behavior. A) Mean distance traveled per day $( \pm \mathrm{SE})$ in the custom-made home cages without wheels plotted separately for each strain. B) Mean distance traveled per day on running wheels $( \pm$ SE) plotted for each strain. The means are collapsed across sex ( $n=8$ animals per bar). The bars are sorted from least active to the most active strain. 

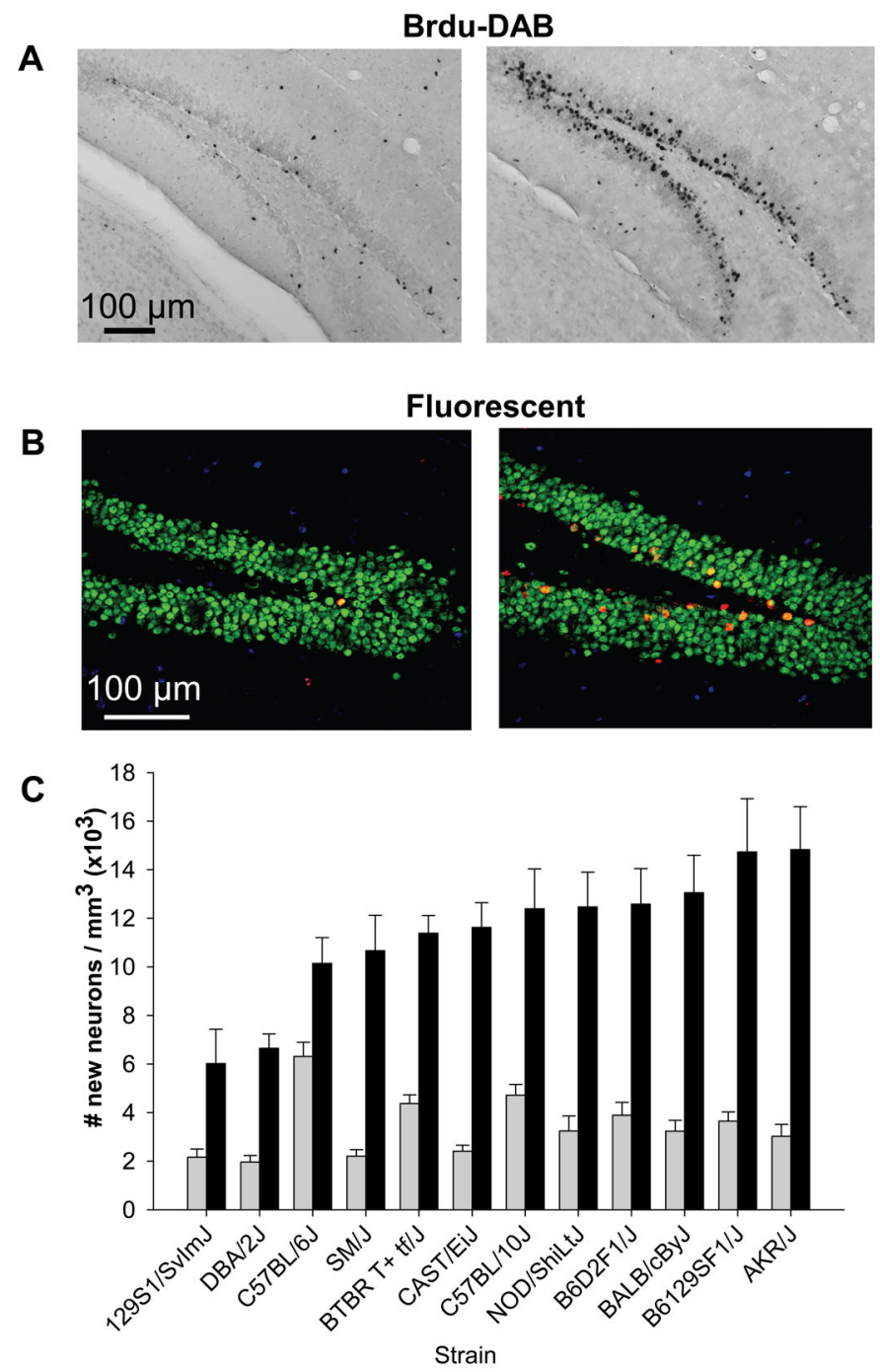

Figure 2.

Genetic differences in exercise-induced adult hippocampal neurogenesis. Top, representative sections through the dentate gyrus of a sedentary mouse and a runner stained for BrdU with A) DAB as the chromogen and B) triple fluorescent stained BrdU red, NeuN (mature neuronal marker) green and S100 $\beta$ (astrocyte marker) blue. C) Mean number of BrdU positive cells per volume granule layer of the dentate gyrus multiplied by the proportion of BrdU cells expressing NeuN $( \pm \mathrm{SE})$ plotted separately for each strain and treatment group (sedentary versus runner), collapsed across sex ( $\mathrm{n}=8$ animals per bar). The bars are sorted from least to most neurogenesis in the runner condition. 


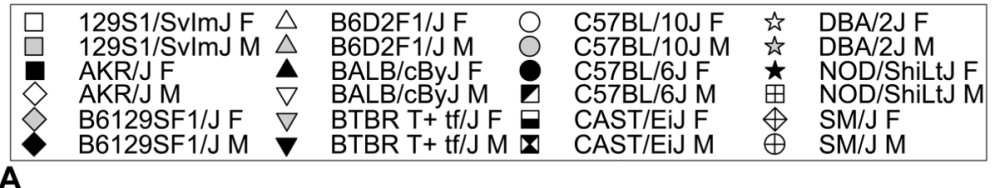

A
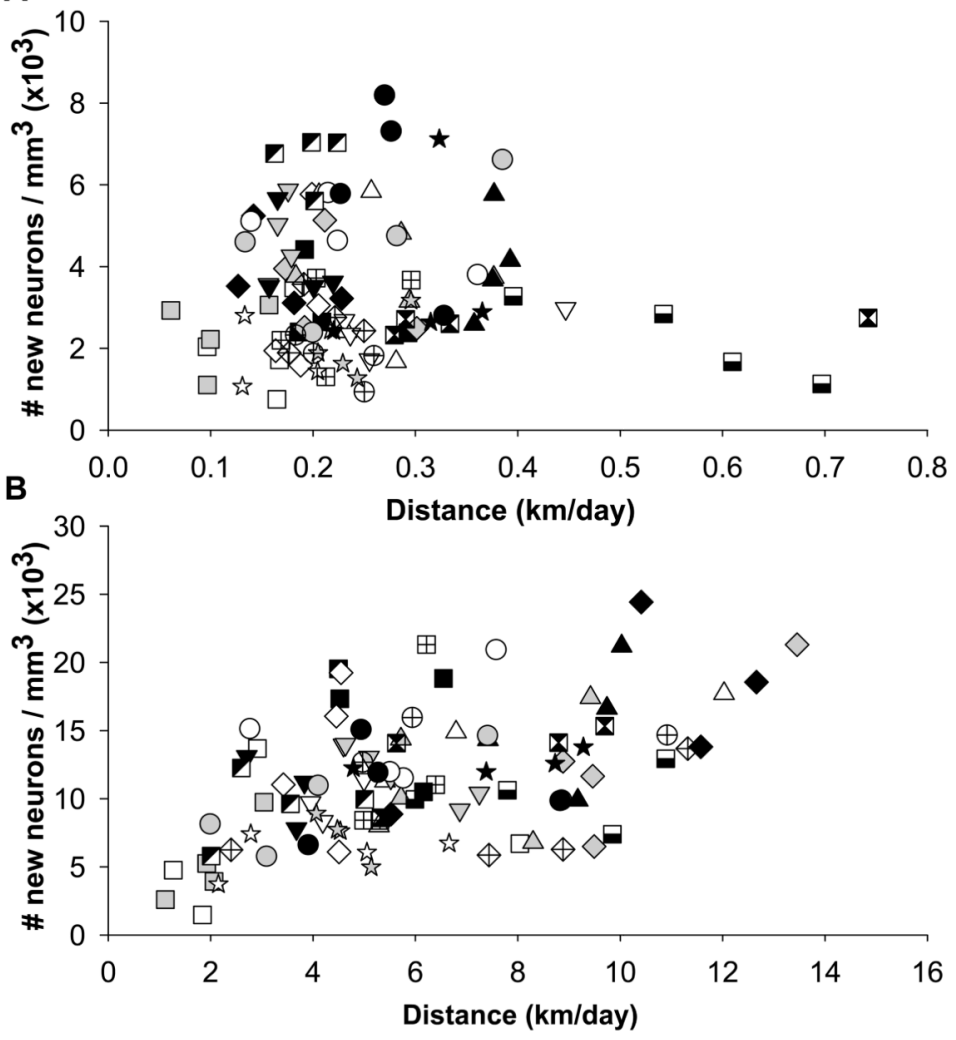

Figure 3.

Analysis of covariance. A) Individual levels of neurogenesis (i.e., number of BrdU positive cells per volume granule layer of the dentate gyrus multiplied by the proportion of BrdU cells expressing NeuN) plotted against distance traveled in cages without wheels. B) Individual levels of neurogenesis plotted against distance run on wheels. 
A

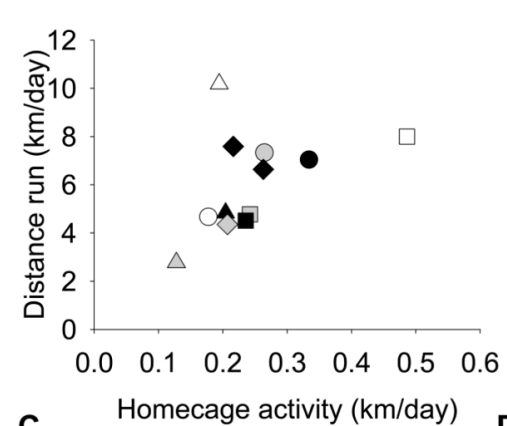

C Homecage activity (km/day)

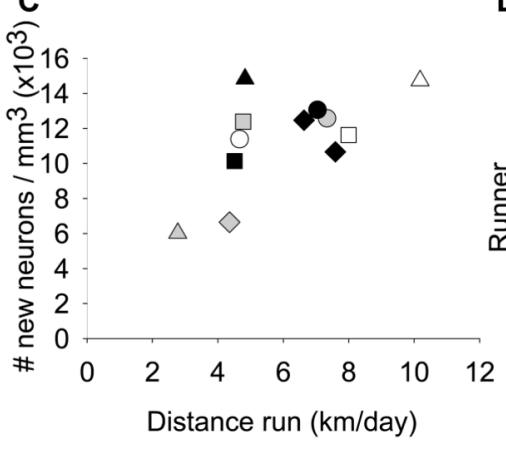

D
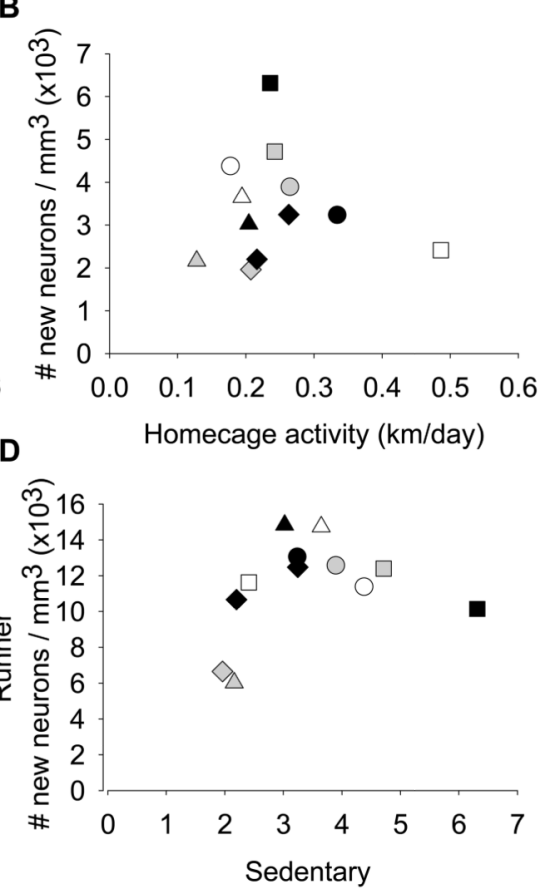

\# new neurons $/ \mathrm{mm}^{3}\left(\times 10^{3}\right)$

129 S1/SvlmJ A AKR/J C57BL/10J B6D2F1/J

DBA/2J

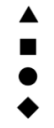

C57BL/6J

BALB/CByJ

NOD/ShiLtJ
B6129SF1/J

CAST/EiJ

BTBR T+ tf/J

SM/J

Figure 4.

Genetic correlations. A) Strain mean distance ( $\mathrm{km} /$ day) accumulated on running wheels plotted against strain mean distance ( $\mathrm{km} /$ day) traveled in sedentary cages without wheels. B) Strain mean level of neurogenesis under sedentary conditions (i.e., mean number of BrdU positive cells per volume granule layer of the dentate gyrus multiplied by the proportion of BrdU cells expressing NeuN) plotted against strain mean distance traveled in cages without wheels. C) Strain mean level of neurogenesis in runners plotted against strain mean distance run on wheels. D) Strain mean level of neurogenesis in runners plotted against strain mean level of neurogenesis under sedentary conditions. 


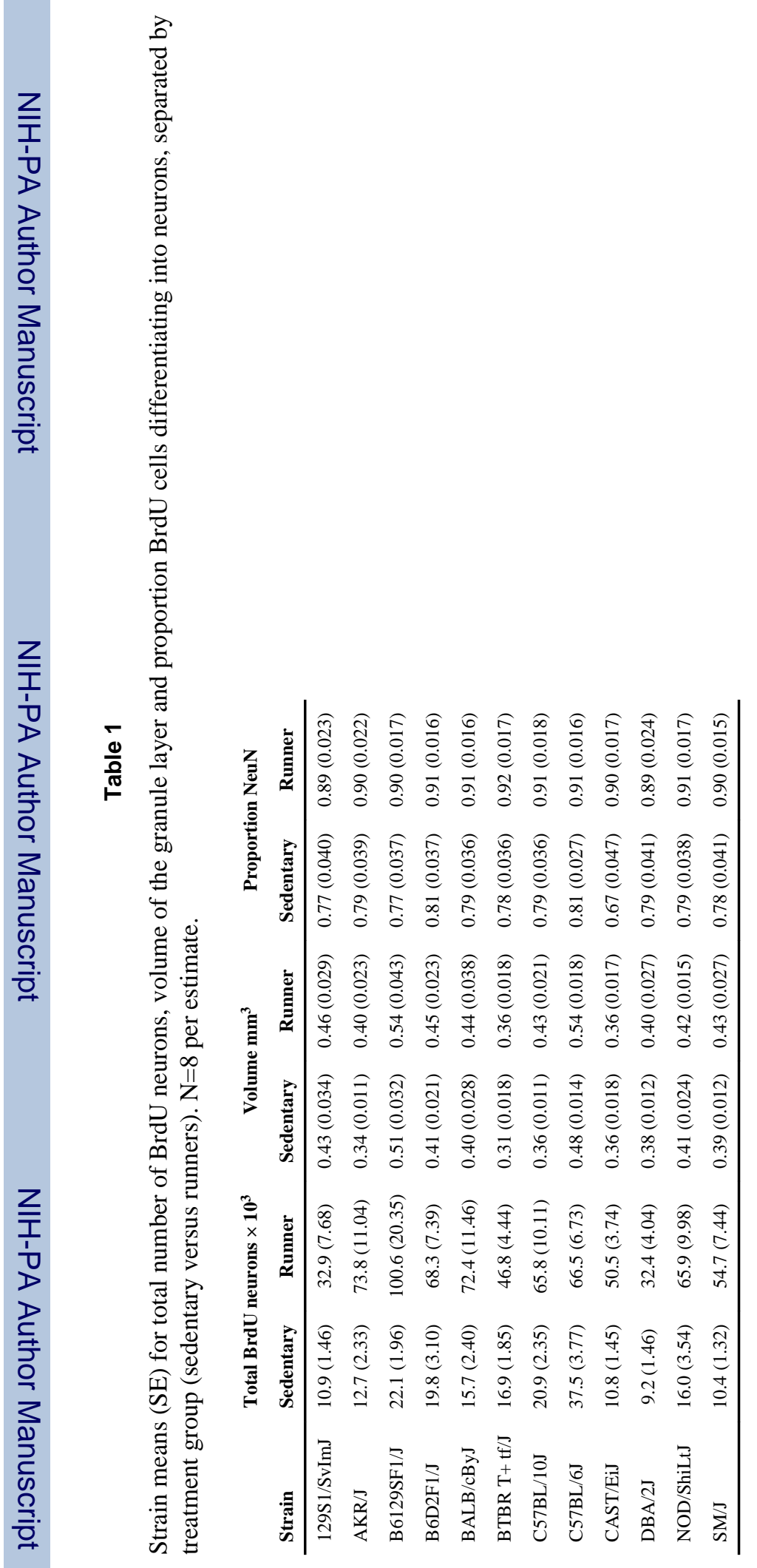




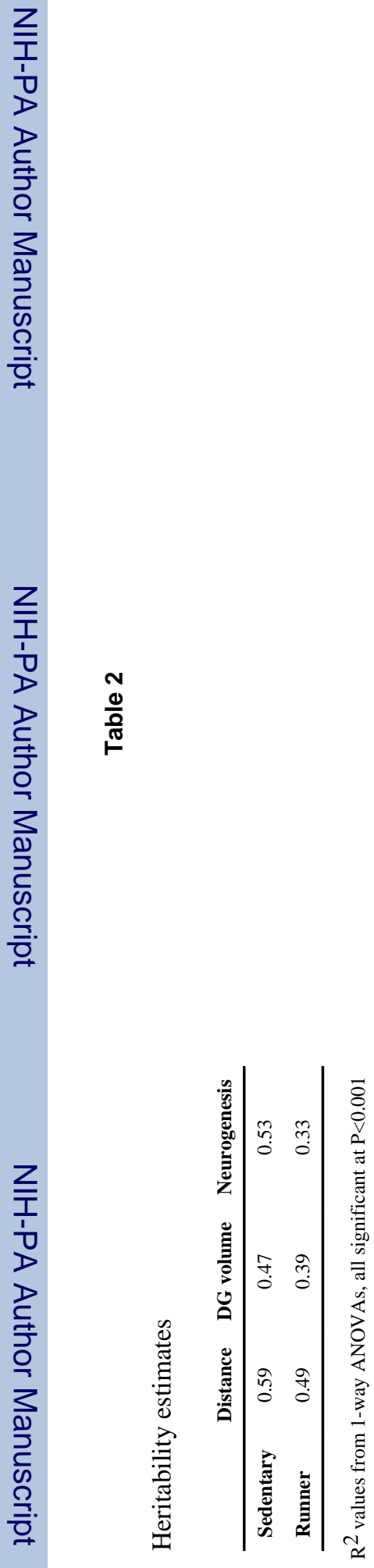

